\title{
The Effect of Physical Activity with Different Levels of Cognitive Load on Executive Control Network
}

\section{of Attention in Youth}

\author{
Mahta Eskandarnejad ${ }^{\mathrm{a}}$, Fahimeh Rezaei ${ }^{\mathrm{b}^{*}}$
}

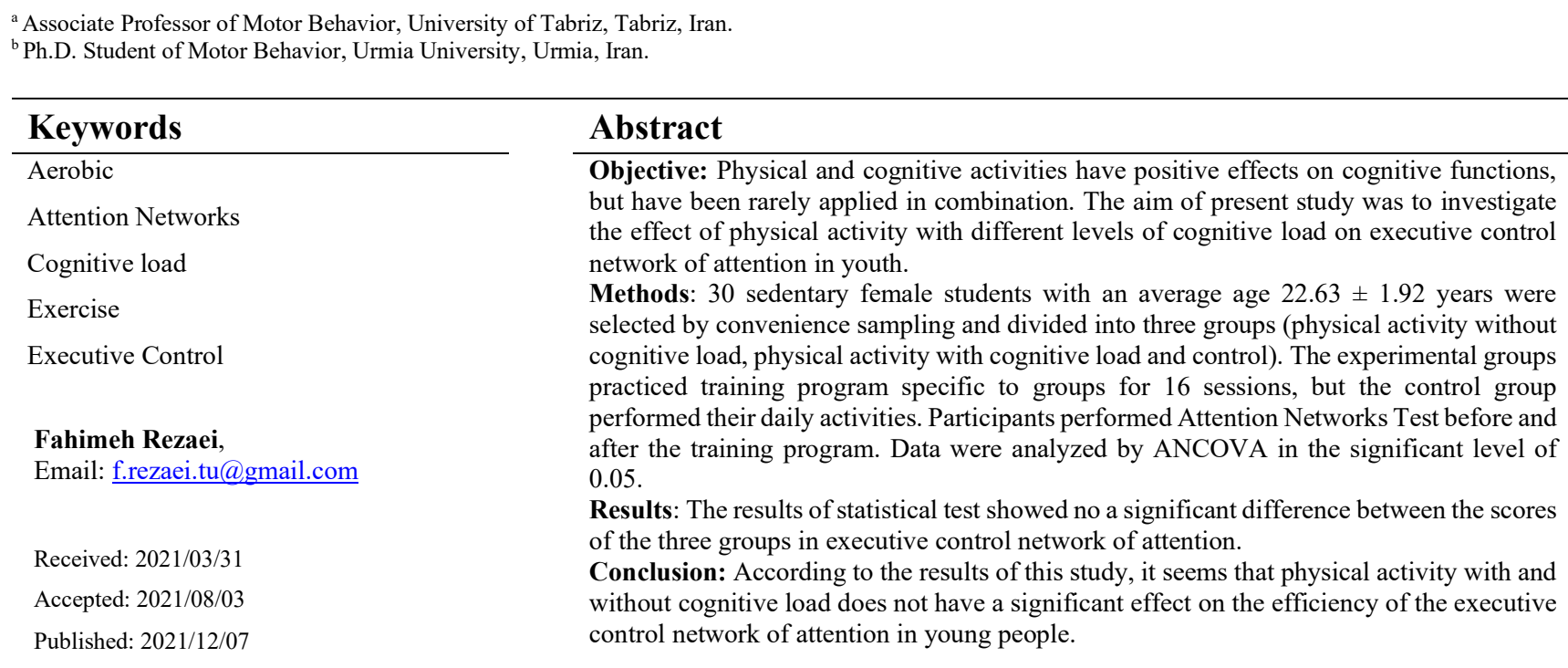

\section{Introduction}

It is now well established that regular exercise promotes a variety of adaptations, including metabolic, cardiovascular, and neuromuscular adaptations) De Vries et al., 2012; Hillman, Erickson, \& Kramer, 2008). Recently, the effects of exercise on the brain and its function have been the focus of much research; New research has shown that numerous molecular anatomical and cellular changes occur as a result of exercise in the central and peripheral nervous system, which ultimately leads to improved cognitive and motor functions (Barari, Bashiri, Rahimi, \& Mokhtari, 2015). One of the important cognitive functions is attention, which is widely introduced as the ability of the mind to focus on a task or subject and is a very important prerequisite for meeting daily demands (Pérez, Padilla, Parmentier, \& Andrés, 2014). Although attention is generally believed to be a general feature of the whole brain, neural imaging studies suggest the existence of specific networks in neural regions that are involved in attention-related functions (Posner, Sheese, Odludaş, \& Tang, 2006). According to the neurological model (Posner \& Petersen, 1990), attention is related to the function of three neural networks: alerting, orienting, and executive control, each of which includes a specific set of 
mental functions and distinct brain regions. In this model, the executive control network refers to the mental actions involved in monitoring, recognizing and resolving conflicts between different brain regions and providing appropriate behavioral responses (Fan et al., 2009; Posner \& Rothbart, 2007) And because it regulates the activity of other brain networks involved with thoughts and emotions, it is called so (Posner et al., 2006). Executive control neural connections include the anterior cingulated cortex and the dorsolateral prefrontal cortex (Fan et al., 2009; Posner \& Rothbart, 2007). The executive control network is modulated by the dopamine neurotransmitter system (Posner \& Rothbart, 2007) Among the diseases associated with network disruption are Alzheimer, borderline personality disorder, schizophrenia, and 22Q11 deletion syndrome (Rothbart \& Posner, 2006). The executive control network is often involved in high-level mental actions and in general with executive functions involved in processes such as planning and performing goal-oriented actions such as working memory, attention flexibility, inhibitory control and conflict monitoring that all of these cognitive abilities and functions are needed when storing, managing, and integrating information into the brain; (Rueda, Checa, \& Cómbita, 2012) therefore, it is very important to study whether the efficiency of this network can be increased by using special methods or not. Interestingly, exercise has recently been proposed as a factor in enhancing the executive control network of attention (Pérez et al., 2014). In a study by (Change, Pesce, Chiang, Kuo, \& Fong, 2015) on the electrical-neural and behavioral parameters of attention networks in young people, it was found that aerobic exercise leads to a larger P3-domain range in the executive control network and It is associated with improving the performance of this network of attention. In contrast, (Huertas, Zahonero, Sanabria, \& Lupiáñez, 2011) study of 18 young adults showed no improvement in executive control network performance after one aerobic exercise session. Recent studies, however, have shown that physical activity combined with cognitive load enhances cognitive abilities beyond what exercise alone can have (Eggenberger, Schumacher, Angst, Theill, \& de Bruin, 2015; Moreau, Morrison, \& Conway, 2015). It has been suggested that physical activity may improve brain metabolism and flexibility, and that a cognitive challenge will increase cognition by increasing brain metabolism and guiding the flexibility process (Rahe et al., 2015). A study (Black, Isaacs, Anderson, Alcantara, \& Greenough, 1990) also showed that animals that did aerobic exercise had a higher density of blood vessels than inactive animals or animals that performed only simple acrobatic movements; In contrast, animals that performed acrobatic movements had more synapses per cerebellar Purkinje cell than animals in the control group and even those in the aerobic exercise group. The researchers suggested that motor learning involving acrobatic movements, unlike aerobic exercise, which requires repeated use of synapses during exercise, develops new synapses in the cerebellum. As a result, it may be possible to take full advantage of the benefits of exercise to improve cognition by combining 
aerobic exercise (greater blood vessel density) and complex movements (increased synapses).

However, a review of the existing research literature shows that despite extensive research on the role of various physical activities in terms of intensity (Maleki, Bahram, Farokhi, Rajabi, \& Yaryari, 2012) and duration (Zeidabadi, Arab Ameri, Naghdi, \& Bolouri, 2014) on cognitive functions, little attention has been paid to the study of sports in terms of cognitive burden. Also, the positive effect of exercise on more cognitive functions in children, the elderly and some clinical populations has been reported (Chaddock, Voss, \& Kramer, 2012; Duchesne et al., 2015) and in contrast to very few studies, its benefits have been studied in young and healthy individuals (Pérez et al., 2014). At the same time, the youth of each country will be responsible for key jobs in the near future and will be considered as the assets of a country; Therefore, the need for planning and investment in their health and giving importance to what strategies can be used to help them in the development of physical and cognitive aspects, is twofold. On the other hand, the executive control network plays a very important role in situations that include planning, decision making, error determination and execution of new responses, and for this reason, it has attracted the most interest of researchers (Fathirezaie, Farsi, Vaani, Zazi, 2015). Therefore, in this study, we tried to investigate the effect of two different types of physical activity in terms of cognitive load on the efficiency of the executive attention control network in young people. The main question that led to this research is whether it is possible to use physical activities with and without cognitive load to improve the executive control network of attention in young people? And is there a difference in the effect of these two types of physical activity on cognition?

\section{Method}

The present study is applied practical in terms of purpose, and in terms of method is semiexperimental with two stages of measurement before and after the training program and the control group.

\section{Participants}

Participants in this study included 30 healthy and sedentary female students of Tabriz University with a mean age of $22.63 \pm 1.92$ years. To collect a research sample, the students who were available were invited to participate in the research if they wished. In a coordination session, after providing the necessary information to those interested in participating in the research, a researcher-made questionnaire was distributed and collected (including questions about personal information, vision status and sports background) and a general health questionnaire (GHQ-12) (with four subscales of physical symptoms, anxiety and insomnia symptoms, social dysfunction symptoms and depressive symptoms with Cronbach's alpha coefficient of 0.92) (Yaghubi, Karimi, Omidi, Barouti, \& Abedi, 2012), 30 people individuals who were eligible for the study were selected as a statistical sample and were divided equally into two experimental groups and one control group. Criteria for participating in the study included general health, normal or corrected vision, and 


\section{Original article}

inactivity and lack of regular and routine sports activities for at least the past two years. Also, the selection of this number of samples for each group was based on the samples of similar studies in the past (Fathirezaie et al., 2015).

\section{Tools}

In this study, in order to investigate the changes in the performance of the executive attention control network, the Attention Network Test (ANT) was used. This software test, developed by (Fan, McCandliss, Sommer, Raz, \& Posner, 2002), is extensively used to measure the efficiency of attention networks by evaluating the reaction time in response to a stimulus visual aid. In this test, flankers are presented as consistent $(>>>>>)$ or inconsistent $(>><>)$ on the computer screen, which by subtracting the reaction time in inconsistent conditions from the reaction time in consistent conditions, the effect of executive control is measured. The attention grid test, which takes about 20 minutes to complete, including the experimental phase, consists of a total of four blocks, the first of which is two minutes long and is for practice and repetition. The other three blocks, which are the main test, each take about six minutes, and a "break" message appears after each block is completed. The test method is that there is a cross $(+)$ in the center of the screen where flankers appear from above or below it. Participants are required to focus on the cross during the test period and with the appearance of the flankers, with high speed and accuracy to the center arrow using the mouse (Left mouse button for when the center arrow point is to the left and right mouse button for when the center arrow point is to the right). The reliability of this test is reported to be 0.87 (Fan et al., 2002).

\section{Data collection method}

Before starting the work, after a complete description of the objectives and method of conducting the research, written consent was obtained from the subjects. The Physical Activity Readiness Questionnaire (PAR-Q) was also distributed and collected to ensure the ability of the participants in the experimental groups to participate and complete the training period. Participants from all three groups were then tested. Then, the participants of both experimental groups underwent two different types of training interventions (physical activity with and without cognitive load) for five weeks (16 sessions). The reason for choosing five weeks is because although it has been shown that the effect of exercise can appear within a week, most responses take longer (3 to 12 weeks) to appear. (Shayan, Bagherzadeh, Shahbazi, \& Choobineh, 2015).

The protocol of physical activity without cognitive load includes an endurance exercise including warm-up and stretching for 10 minutes and then walking and running with an intensity of $70-85 \%$ of maximum heart rate for 40 minutes in two consecutive sets of 20 minutes with a five-minute break between sets. In this exercise program, the subject under the control of the examiner did stretching movements for 10 minutes and then started running. The subject was told that based on the heart rate range (estimated based on the individual's maximum heart rate $\{220$-age $\}$ ) 
(Pescatello, Riebe, \& Thompson, 2014) assigned to him and through the pacemaker attached to his body (Ceo537 Polar Belt and Timer, which was fastened to the chest and wrist) has continued to run, so that his heart rate does not fall below $70 \%$ and does not exceed $85 \%$. At the end, cooling for five minutes was considered.

The protocol of physical activity with cognitive load also included aerobic chain training. In this study, participants started the exercise from simpler chains performed by an experienced instructor and gradually added to the complexity of the chains. To determine the intensity of training in the aerobic group and in order to match it with another experimental group, the number of beats per minute or BPM of music was used. In aerobic classes, to keep the heart rate within the aerobic range ( 70 to $85 \%$ of maximum heart rate), the rhythm of the music should be in the range of 110 to 120 (BPM), and the chains taught in the previous sessions should be repeated with a constant rhythm (Eskandarnejad \& Ashkriz, 2018). The duration of the protocol was 60 minutes, which included 10 minutes of warm-up and stretching, 45 minutes of aerobic chain training, and the last five minutes of cooling down.

Finally, the post-test was performed at least 48 hours after the last training session in order to remove the temporary effects of training from the participants (Shayan et al., 2015).

\section{Data analysis}

Finally, descriptive statistics were used to describe and classify the raw scores through the calculation of mean and standard deviation, and in the inferential statistics section, one-way analysis of covariance was used to test the research hypothesis. All analyzes were performed at a significant level of 0.05 using SPSS version 20 software.

\section{Results}

Data related to statistical description are presented in Table 1.

Table 1: Data related to statistical description.

\begin{tabular}{lllll}
\hline \multicolumn{1}{c}{ Group } & \multirow{2}{*}{ Number } & \multirow{2}{*}{ Age (years) } & $\begin{array}{c}\text { Executive control network (time) } \\
\text { Post-test }\end{array}$ \\
$\begin{array}{lllll}\text { Variable } & & & & \text { Pre-test }\end{array}$ \\
$\begin{array}{l}\text { Physical activity without cognitive } \\
\text { load }\end{array}$ & 10 & $2.07 \pm 22.1$ & $50.86 \pm 160.12$ & $31 \pm 108.25$ \\
$\begin{array}{l}\text { Physical activity with cognitive load } \\
\text { Control }\end{array}$ & 10 & $1.43 \pm 22.5$ & $45.2 \pm 128.55$ & $27.85 \pm 102.11$ \\
\hline
\end{tabular}

Before performing statistical analysis, ShapiroWilk test was used to check the normality of data distribution. After confirming the normality of the data $(\mathrm{P}>0.05)$, independent t-test was used to check the presence or absence of differences in the pre-tests. Then, due to the significant difference in the pre-test of the three groups $(\mathrm{P}<0.05)$, to analyze the data of this study, the statistical method of oneway analysis of covariance in which the pre-test scores as a co-diffraction variable used to control previous differences between groups. First, preliminary studies were performed to ensure that the other hypotheses of this statistical method were not violated and the assumption of equality of 
variances was confirmed using Levin test $(\mathrm{P}>$ 0.05). In order to investigate the assumption of regression slope homogeneity, the interaction effect of co-diffraction variable with the group was evaluated, which due to its insignificance $(\mathrm{P}=$
$0.33)$, the regression homogeneity assumption was also realized. With all the assumptions fulfilled, the one-way analysis of covariance was performed, the results of which are shown in Table 2.

Table 2: Summary of the results of analysis of covariance to compare the scores of the executive control network of attention in the study groups.

\begin{tabular}{lllllll}
\hline Source & $\begin{array}{l}\text { Sum of } \\
\text { Squares }\end{array}$ & df & $\begin{array}{l}\text { Mean } \\
\text { Square }\end{array}$ & F & Sig & $\begin{array}{l}\text { Partial Eta } \\
\text { Squared }\end{array}$ \\
\hline Pre-test & 10842.99 & 1 & 10842.99 & 29.82 & 0.001 & 0.611 \\
Group & 615.38 & 2 & 307.69 & 0.846 & 0.445 & 0.082 \\
Error & 6908.22 & 26 & 363.59 & & & \\
\hline
\end{tabular}

The results of Table 2 show that after adjusting the pre-test scores, the experimental groups did not notice a statistically significant difference in the efficiency of the executive control network compared to the control group $(\mathrm{P}=0.445)$.

\section{Discussion and Conclusion}

The aim of this study was to investigate the effect of physical activity with different levels of cognitive load on the efficiency of the neural network of executive attention control in young people. The results of this study showed that there was no change in the performance of the executive attention control network after performing physical activity without cognitive load. Consistent with the findings of this study, we can refer to the results of the study (Huertas et al., 2011) that, like this study, did not observe a statistically significant change in the efficiency of this neural network after physical activity. It has been reported that the effectiveness of exercise may be selective on cognition and may depend on the nature of cognitive functions and the brainstems associated with them (Boucard et al.,
2012). In a study conducted by (Eskandarnejad \& Rezaei, 2018), the results showed that aerobic exercise improved working memory, but did not have a significant effect on the positioning network. In the study (Boucard et al., 2012) the effect of physical activity on the inhibition variable was significant, but no significant updating and shifting was observed in both variables. However (Weinstein et al., 2012) in a study showed that a high level of physical fitness is associated with a high volume of gray matter in the dorsolateral prefrontal cortex, which is one of the important areas involved with the executive control network. In particular, neuroimaging studies have reported greater prefrontal area and white matter and gray matter in the anterior part of the brain as characteristics of active individuals (Colcombe et al., 2004; Flöel et al., 2010; Gordon et al., 2008), which is typically related to the executive control network of attention (Fan et al., 2009; Posner \& Rothbart, 2007). However, a study by (Hillman et al., 2006) found that aerobic exercise reduced the negative effects of dissonant stimuli only in the 
elderly and not in the young. According to the moderating model proposed by (Stones \& Kozma, 1988), it has been hypothesized that the benefits of exercise on cognitive function should be enhanced with age. In this regard, some studies have reported in the early 60s (Bunce \& Murden, 2006) and some after the 70s (Renaud, Bherer, \& Maquestiaux, 2010) that the positive effect of physical activity on cognitive functions in these ages are stronger. In this regard, in a study conducted by (Boucard et al., 2012) to investigate the relationship between cardiovascular fitness and executive functions of people aged in three groups of 18 to 28 years, 60 to 70 years and 71 to 83 years, showed that the positive effect of cardiovascular fitness was particularly significant only in people 71 to 83 years and in the other two age groups there was no difference between active and inactive groups in executive functions. The results of research (Hopkins, Nitecki, \& Bucci, 2011) also showed that the effect of exercise on the brain and nervous system is age-dependent. The researchers reported that there was no significant difference in the amount of brain-derived neurotrophic factor (BDNF) between the training and control groups in adolescence, but this difference is evident in adulthood. Studies in animal models have also shown that elderly or brain-injured animals show the potential effects of physical activity in alleviating nervous system dysfunction (Zeidabadi et al., 2014). In fact, older people have limited cognitive resources and less cognitive ability than younger people, which increases their capacity to improve cognitive function through physical activity (Salthouse \& Davis, 2006) by preventing cognitive decline. (Hillman et al., 2006), decrease in age-related atrophy in gray and white matter, higher density of gray matter in front of brain and temporomandibular region (Gordon et al., 2008), high density of white matter in corpus callosum (Johnson, Kim, Clasey, Bailey, \& Gold, 2012) and create synaptic and cognitive flexibility (Vaynman \& Gomez - Pinilla, 2006). Research has also shown that children with low inhibitory control capacity may benefit more from exercise sessions to improve cognitive function (Drollette et al., 2014). Exercise also benefits most young people with generally low cognitive functions, so the effect of exercise on cognition is not the same for everyone (Sibley \& Beilock, 2007). In fact, healthy and young people have cognitive abilities, so the observation of increased cognitive functions using physical and quantitative interventions in them is much less compared to the elderly and clinical examples (Salthouse \& Davis, 2006). Therefore, the discrepancy between the findings of this study and the results of the study (Colcombe et al., 2004) which reported the positive effect of physical activity on the executive attention control network in the elderly, can be attributed to some extent to the difference in the calendar age and cognitive capacity of the participants of this study with the participants of the mentioned study. On the other hand, (Pérez et al., 2014) conducted a study on young people and concluded that chronic aerobic exercise improves the efficiency of the executive control network, which is not consistent with the findings of the present study. This discrepancy can also be attributed to differences in the method and conduct of research. This is because the study 
compared active people who did aerobic exercise for at least 10 years and at least three days a week with inactive people, but in the present study, the participants were sedentary people who only were engaged in regular physical activity for 5 weeks. It has been reported that by participating in long-term exercise sessions, more stable changes in cognitive processes may occur (Sadeghi, Khalaji, Nourozian, \& Mokhtari, 2013). Also, the reasons for the existence of contradictory results in the research literature regarding the effectiveness of exercise training on cognitive functions have been attributed to some extent to differences in complexity and sensitivity and method of answering tasks and cognitive tests (Pérez et al., 2014). The results of the study (Li et al., 2014) support this claim. Another finding of this study showed that physical activity with cognitive load did not affect the efficiency of the executive attention control network in young people. This finding is consistent with the results of research (Chang et al., 2017) and inconsistent with the results of research (Fathirezaie et al., 2015). In the study (Fathirezaie et al., 2015), participants were divided into experimental and control groups and performed table tennis exercises, with the difference that the experimental group combined table tennis movements with cognitive tasks with working memory load. they did. Therefore, the inconsistency of the results of this research with the mentioned research can be attributed to the difference in some methodology variables of research such as the type of training protocol used. Also, the results of the study (Barcelos et al., 2015) are inconsistent with the results of this study. In this study, elderly people with an average age of 82 years on a workhorse, while for one group was assigned a task with low cognitive demand and for the other group a task with high cognitive demand, was engaged in activity for three months and were evaluated by Stroop test. Apart from the training protocol, the reasons for the inconsistency of the results of the present study with the mentioned study are the differences in the number of training sessions, the type of test used and the calendar age of the research participants.

In this study, in addition to examining the effect of individual physical exercises with and without cognitive load on the executive attention control network, the effect of these two training programs on cognition was compared, and statistical results showed a significant difference between the effectiveness of these two types of sports interventions on the executive control network has not been considered. Consistent with the results of this study, we can refer to the researchers conducted by (Chang et al., 2017) and (Van den Berg et al., 2016) which indicate that there is no difference between the effectiveness of physical exercises with different levels of cognitive load on the cognitive functions of young people, especially attention. However, studies such as research (Moreau et al., 2015) have shown that the interaction of physical exercises with cognitive challenge leads to improved cognition. On the other hand, research (Tang, 2013) showed the best performance in both immediate and delayed recall in terms of physical activity alone (walking) and recall score when physical activity was combined with cognitive activity (walking-puzzle) reduced. 
Therefore, it seems that the change in cognitive functions varies depending on the type of physical intervention, and because different training patterns and protocols are used in research, thus allowing a complete comparison and a general conclusion about the effects of physical activity on cognitive functions has not yet been possible; It is suggested to refer to meta-analysis studies in this field (Du et al., 2018; Vazou, Pesce, Lakes, \& Smiley-Oyen, 2019).

Finally, although the findings of this study indicate that there is no significant effect of physical activity with different levels of cognitive burden on the efficiency of executive attention control network in young people, nevertheless, the beneficial effects of physical activity on cognition cannot be ignored. Therefore, it is suggested that in similar studies, the effect of physical exercise on different cognitive functions in different age groups be compared with different cognitive tests. It is also suggested that neuro-physiological evidence and neuropsychological involvement for the functioning of the executive attention control network following different physical activities be examined to provide appropriate explanations of the function and the structure of the cerebral cortex should be provided to researchers in response to these types of activities. Among the limitations of the present study were genetic differences and the level of motivation and mental involvement of research participants during the research test, which was beyond the control of the researcher.

\section{Conflict of interest}

The authors declare that there is no conflict of interest.

\section{References}

1. Barari, A. R., Bashiri, J., Rahimi, A. R., \& Mokhtari, E. (2015). The effect of endurance and circuit resistance training on serum brainderived neurotrophic factor and cortisol in inactive male students: A randomized clinical trial. Journal of ShahrekordUuniversity of Medical Sciences, 17(2), 43-53.

2. Barcelos, N., Shah, N., Cohen, K., Hogan, M. J., Mulkerrin, E., Arciero, P. J., . . . AndersonHanley, C. (2015). Aerobic and Cognitive Exercise (ACE) pilot study for older adults: executive function improves with cognitive challenge while exergaming. Journal of the International Neuropsychological Society, 21(10), 768-779.

3. Black, J. E., Isaacs, K. R., Anderson, B. J., Alcantara, A. A., \& Greenough, W. T. (1990). Learning causes synaptogenesis, whereas motor activity causes angiogenesis, in cerebellar cortex of adult rats. Proceedings of the National Academy of Sciences, 87(14), 5568-5572.

4. Boucard, G. K., Albinet, C. T., Bugaiska, A., Bouquet, C. A., Clarys, D., \& Audiffren, M. (2012). Impact of physicalactivity on executive functions in aging: a selective effect on inhibition among old adults. Journal of Sport and Exercise Psychology, 34(6), 808-827.

5. Bunce, D., \& Murden, F. (2006). Age, aerobic fitness, executive function, and episodic memory. EuropeanJournal of Cognitive Psychology, 18(2), 221-233.

6. Chaddock, L., Voss, M. W., \& Kramer, A. F. (2012). Physical activity and fitness effects on cognition and brain health in children and older adults. Kinesiology Review, 1(1), 37-45.

7. Chang, E., Chu, C., Karageorghis, C. I., Wang, C., Tsai, J., Wang, Y., \& Chang, Y. (2017). Relationship between mode of sport training and general cognitive performance. Journal of Sport and Health Science, 6(1), 89-95.

8. Change, Y., Pesce, C., Chiang, Y., Kuo, C., \& Fong, D. (2015).Antecedent acute cycling exercise affects attention control: an ERP study using attention network test. Frontiers in human neuroscience, 9, 156.

9. Colcombe, S. J., Kramer, A. F., Erickson, K. I., Scalf, P., McAuley, E., Cohen, N. J., . . . 
Elavsky, S. (2004). Cardiovascular fitness, cortical plasticity, and aging. Proceedings of the National Academy of Sciences, 101(9), 3316-3321.

10. De Vries, N., Van Ravensberg, C., Hobbelen, J., Rikkert, M. O., Staal, J., \& Nijhuis-Van der Sanden, M. (2012). Effects of physical exercise therapy on mobility, physical functioning, physical activity and quality of life in community-dwelling older adults with impaired mobility, physical disability and/or multi-morbidity: a meta-analysis. Ageing research reviews, 11(1), 136-149.

11. Drollette, E. S., Scudder, M. R., Raine, L. B., Moore, R. D., Saliba, B. J., Pontifex, M. B., \& Hillman, C. H. (2014). Acute exercise facilitates brain function and cognition in children who need it most: an ERP study of individual differencesin inhibitory control capacity. Developmental cognitive neuroscience, 7, 53-64.

12. Du, Z., Li, Y., Li, J., Zhou, C., Li, F., \& Yang, $X$. (2018). Physical activity can improve cognition in patients with Alzheimer's disease: a systematic review and meta-analysis of randomized controlled trials. Clinical interventions in aging, 13, 1593-1603.

13. Duchesne, C., Lungu, O., Nadeau, A., Robillard, M., Boré, A., Bobeuf, F., ... Doyon, J. (2015). Enhancing both motor and cognitive functioning in Parkinson's disease: aerobic exercise as a rehabilitative intervention. Brain and cognition, 99, 68-77.

14. Eggenberger, P., Schumacher, V., Angst, M., Theill, N., \& de Bruin, E. D. (2015). Does multicomponent physical exercise with simultaneous cognitive training boost cognitiveperformance in older adults? A 6month randomized controlled trial with a 1 -year follow-up. Clinical interventions in aging, 10, 1335-1349.

15. Eskandarnejad, M., \& Ashkriz, N. (2018). Learning Step by Step of Aerobics and stepp. Tabriz: University of Tabriz.

16. Eskandarnejad, M., \& Rezaei, F. (2018). The Effect of Aerobic Exercise on Neural Networks of Attention and Working Memory. The Neuroscience Journal of Shefaye Khatam, 6(2), 31-40.

17. Fan, J., Gu, X., Guise, K. G., Liu, X., Fossella, J., Wang, H., \& Posner, M. I. (2009). Testing the behavioral interaction and integration of attentional networks. Brain and cognition, 70(2), 209-220.

18. Fan, J., McCandliss, B. D., Sommer, T., Raz, A., \& Posner, M. I. (2002). Testing the efficiency and independence of attentional networks. Journal of cognitive neuroscience, 14(3), 340-347.

19. Fathirezaie, Z., Farsi, A., VaezMousavi, M. K., \& ZamaniSani, S. H. (2015). Effect of cognitive training on efficiency of executive control network of attention. Journal of Research in Rehabilitation Sciences, 11(3), 182-192.

20. Flöel, A., Ruscheweyh, R., Krüger, K., Willemer, C., Winter, B., Völker, K., . . . Breitenstein, C. (2010). Physical activity and memory functions: are neurotrophins and cerebral gray matter volume the missing link? Neuroimage, 49(3), 2756-2763.

21. Gordon, B. A., Rykhlevskaia, E. I., Brumback, C. R., Lee, Y., Elavsky, S., Konopack, J. F., . . . Gratton, G. (2008). Neuroanatomical correlates of aging, cardiopulmonary fitness level, and education. Psychophysiology, 45(5), 825-838.

22. Hillman, C. H., Erickson, K. I., \& Kramer, A. F. (2008). Be smart, exercise your heart: exercise effects on brain and cognition. Nature reviews neuroscience, 9(1), 58-65.

23. Hillman, C. H., Motl, R. W., Pontifex, M. B., Posthuma, D., Stubbe, J. H., Boomsma, D. I., \& De Geus, E. J. (2006). Physical activity and cognitive function in a cross-section of younger and older community-dwelling individuals. Health psychology, 25(6), 678-687.

24. Hopkins, M. E., Nitecki, R., \& Bucci, D. J. (2011). Physical exercise during adolescence versus adulthood: differential effects on object recognition memory and brain-derived neurotrophic factor levels. Neuroscience, 194, 84-94.

25. Huertas, F., Zahonero, J., Sanabria, D., \& Lupiáñez, J. (2011). Functioning of the attentional networks at rest vs. during acute bouts of aerobic exercise. Journal of Sport and Exercise Psychology, 33(5), 649-665.

26. Johnson, N. F., Kim, C., Clasey, J. L., Bailey, A., \& Gold, B. T. (2012). Cardiorespiratory fitness is positively correlated with cerebral white matter integrity in healthy seniors. Neuroimage, 59(2), 1514-1523.

27. Li, L., Men, W.-W., Chang, Y.-K., Fan, M.-X., Ji, L., \& Wei, G.-X. (2014). Acute aerobic exercise increases cortical activity during working memory: a functional MRI study in female college students. PloS one, 9(6), e99222.

28. Maleki, M., Bahram, A., Farokhi, A., Rajabi, H., \& Yaryari, F. (2012). The Effect of Different Intensities of Aerobic Physical Exercise on the Performance of Visual Choice Reaction Time and Stroop Test. Journal of Research in Psychological Health, 5(3), 1-12. 
29. Moreau, D., Morrison, A. B., \& Conway, A. R. (2015). An ecological approach to cognitive enhancement: complex motor training. Acta psychologica, 157, 44-55.

30. Pérez, L., Padilla, C., Parmentier, F. B., \& Andrés, P. (2014). The effects of chronic exercise on attentional networks. PLoS One, 9(7), e101478.

31. Pescatello, L. S., Riebe, D., \& Thompson, P. D. (2014). ACSM's guidelines for exercise testing and prescription. NewYork: Lippincott Williams\&Wilkins.

32. Posner, M. I., \& Petersen, S. E. (1990). The attention system of the human brain. Annual review of neuroscience, 13(1), 25-42.

33. Posner, M. I., \& Rothbart, M. K. (2007). Research on attention networks as a model for the integration of psychological science. Annual Review of Psychology, 58, 1-23.

34. Posner, M. I., Sheese, B. E., Odludaş, Y., \& Tang, Y. (2006). Analyzing and shaping human attentional networks. Neural networks, 19(9), 1422-1429.

35. Rahe, J., Becker, J., Fink, G. R., Kessler, J., Kukolja, J., Rahn, A., . . . Kalbe, E. (2015). Cognitive training with and without additional physical activity in healthy older adults: cognitive effects, neurobiological mechanisms, and prediction of training success. Frontiers in aging neuroscience, 7, 187.

36. Renaud, M., Bherer, L., \& Maquestiaux, F. (2010). A high level of physical fitness is associated with more efficient response preparation in older adults. Journals of Gerontology Series B: Psychological Sciences and Social Sciences, 65(3), 317-322.

37. Rothbart, M. K., \& Posner, M. I. (2006). Temperament, attention, and developmental psychopathology. In Handbook of Developmental Psychopathology. New York: Wiley.

38. Rueda, M. R., Checa, P., \& Cómbita, L. M. (2012). Enhanced efficiency of the executive attentionnetwork after training in preschool children: immediate changes and effects after two months. Developmental cognitive neuroscience, 2, 192-204.

39. Sadeghi, N., Khalaji, H., Nourozian, M., \& Mokhtari, P. (2013). The impact of physical activity on the memory of women 50-70 years old with memory impairment. New Cellular and Molecular Biotechnology Journal, 3(11), 47-54.

40. Salthouse, T. A., \& Davis, H. P. (2006). Organization of cognitive abilities and neuropsychological variables across the lifespan. Developmental Review, 26(1), 31-54.
41. Shayan, A., Bagherzadeh, F., Shahbazi, M., \& Choobineh, S. (2015). The effect of two types of exercise (endurance and resistance) on attention and brain derived neurotropic factor levels in sedentary students. Journal of Motor Learning and Movement, 6(4), 433-452.

42. Sibley, B. A., \& Beilock, S. L. (2007). Exercise and working memory: an individual differences investigation. Journal of Sport and Exercise Psychology, 29(6), 783-791.

43. Stones, M. J., \& Kozma, A. (1988). Physical activity, age, and cognitive/motor performance In L. Howe \& C.J. Brainerd (Eds.), Cognitive Development in Adulthood (pp. 271-321). New York: Springer-Verlag.

44. Tang, Y. (2013). Post-Learning activities and memory consolidation: the effect of physical and cognitive activities on memory consolidation[dissertation]. Oberlin College, Ohio.

45. Van den Berg, V., Saliasi, E., de Groot, R. H., Jolles, J., Chinapaw, M. J., \& Singh, A. S. (2016). Physical activity in the school setting: Cognitive performance is not affected by three different types of acute exercise. Frontiers in Psychology, 7, 723.

46. Vaynman, S., \& Gomez-Pinilla, F. (2006). Revenge of the "sit": how lifestyle impacts neuronal and cognitive health through molecular systems that interface energy metabolism with neuronal plasticity. Journal of neuroscience research, 84(4), 699-715.

47. Vazou, S., Pesce, C., Lakes, K., \& SmileyOyen, A. (2019). More than one road leads to Rome: a narrative review and meta-analysis of physical activity intervention effects on cognition in youth. International Journal of Sport and Exercise Psychology, 17(2), 153178.

48. Weinstein, A. M., Voss, M. W., Prakash, R. S., Chaddock, L., Szabo, A., White, S. M., .. . Kramer, A. F. (2012). The association between aerobic fitness and executive function is mediated by prefrontal cortex volume. Brain, behavior, and immunity, 26(5), 811-819.

49. Yaghubi, H., Karimi, M., Omidi, A., Barouti, E., \& Abedi, M. (2012). Validity and Factor Structure $f$ the General Health Questionnaire (GHQ-12) In University Students. International Journal Of Behavioral Sciences, 6(2), 153-160.

50. Zeidabadi, R., Arab Ameri, E., Naghdi, N., \& Bolouri, B. (2014). The effect of low- intensity short and long term physical activity on spatial learning and memory in rat. Motor Behavior, 6(15), 155-172. 